\title{
Multiple-Cytokine Profiling: A Novel Method for Early Prediction of the Efficacy of Sublingual Immunotherapy in Allergic Rhinitis Patients
}

\author{
Yanni Zhang (D), Kang Zhu, Cui Xia, Jingguo Chen, Chao Yu, Tianxi Gao, Jing Yan (D), Huihui Zhang, \\ Xiaoyong Ren \\ Department of Otolaryngology-Head and Neck Surgery, Second Affiliated Hospital of Xi'an Jiaotong University, Xi'an, Shaanxi, People's Republic of \\ China \\ Correspondence: Xiaoyong Ren, Email renxiaoyong@vip.sina.com
}

Background: Allergic rhinitis (AR) is a common inflammatory airway disease, and allergen-specific immunotherapy (AIT) is the only disease-modifying treatment for it. However, not all AR patients respond to AIT, and early prediction of patient response is extremely important. This study aimed to example serum levels of multiple cytokines in AR and explore their association with the efficacy of AIT.

Methods: A total of 74 AR patients treated with sublingual immunotherapy (SLIT) were prospectively recruited. Serum samples were obtained before the onset of SLIT and cytokine levels detected by multiplex analysis. All patients were followed for $>1$ year, and associations between cytokine levels and the early efficacy of SLIT were evaluated. Significantly distinctive cytokines were further verified in another independent cohort.

Results: Sixty patients completed the visit schedule set: 35 patients were put into a responder group and 25 a nonresponder group. Multiple-cytokine profiling showed that cytokine levels differed significantly between the two groups. The responder group had higher concentrations of BAFF and CCL11 and lower levels of CCL2, CCL7, IFN $\gamma$, IL8, IL10, IL16, and IL33 than the nonresponder group $(P<0.05)$. Receiver-operating characteristic curves highlighted that serum BAFF, IFN $\gamma$, IL10, and IL33 levels were strongly predictive of the efficacy of SLIT (area under the curve $<0.7, P<0.05$ ). Serum IL10 and IL33 were overexpressed in nonresponders in the validation cohort. Patients in the responder group exhibited significantly higher IL10 levels and lower IL33 post-SLIT than pre-SLIT $(P<0.05)$, but no statistical difference was found in nonresponders $(P<0.05)$.

Conclusion: Our data indicated that serum multiple-cytokine profiling was associated with response to SLIT and that IL10 and IL33 might serve as novel biomarkers for early prediction of efficacy and be involved in the therapeutic mechanisms of SLIT in AR patients. Keywords: allergic rhinitis, sublingual immunotherapy, multiple cytokines, efficacy

\section{Introduction}

Allergic rhinitis (AR) is a common upper-airway disease that is characterized by IgE-mediated $\mathrm{T}_{\mathrm{h}} 2$ inflammation. ${ }^{1,2}$ Recently, epidemiological studies have revealed that AR affects $20 \%-30 \%$ of the population worldwide, and its prevalence continues to increase. ${ }^{3,4}$ It has been recognized that AR has severe adverse effects on daily life, work, and study and increases the risk of asthma, allergic conjunctivitis, and chronic sinusitis. ${ }^{5-7}$ Currently, allergen avoidance, medications, and allergen-specific immunotherapy (AIT) are the mainstays of AR treatment, and AIT is the only diseasemodifying treatment that can induce allergen tolerance. ${ }^{8-10}$ Conventional AIT can be performed subcutaneously or sublingually, and sublingual immunotherapy (SLIT) seems to be more popular because of its convenience, equivalent efficacy, and good tolerability. ${ }^{11-13}$ Despite its widely accepted safety and effectiveness in AR patients, many users still respond poorly, and its effectiveness fluctuates widely. ${ }^{9}$ Therefore, exploring biomarkers or methods for early identification of responders, monitor therapeutic effects in AR patients who have been assigned to SLIT is a research focus, and extremely pivotal for developing a precision medicine model and reducing the waste of medical resources. 
Although prior studies have identified several potential biomarkers to objectively predict the effectiveness of SLIT, including serum-specific IgE, ${ }^{14} \mathrm{MIF},{ }^{15}$ metabolites, ${ }^{16}$ and vitamin $\mathrm{D},{ }^{17}$ these indicators are not clinically viable because of their poor sensitivity, specificity, and repeatability. At present, there is a lack of knowledge concerning the expression of multiple serum cytokines in AR patients and their associations with the efficacy of SLIT. We thus sought to example multiple serumcytokine levels in AR and explore their association with the effectiveness of SLIT.

\section{Methods}

\section{Participants and Setting}

A total of 74 AR patients who visited our department and underwent SLIT between May 2020 and July 2020 were prospectively included in this study. All patients met the inclusion criteria of AR diagnosis as per Allergic Rhinitis and its Impact on Asthma (ARIA) guidelines, ${ }^{18}$ aged 18-60 years old, skin tests positive for Dermatophagoides farinae and/or $D$. pteronyssinus $(\geq++)$ and/or specific $\mathrm{IgE}(>0.35 \mathrm{IU} / \mathrm{mL})$, and symptoms typical of $\mathrm{AR}$ for $>3$ years. We excluded those with other immunologic or inflammatory disease, a history of immunotherapy, pregnant or potentially pregnant, and systemic steroid/antiallergy-medication consumption within the $4 \mathrm{r}$ weeks prior to enrollment. Routine examinations were performed, and demographic and clinical data were collected before the onset of SLIT. The Human Ethics Committee of the Second Affiliated Hospital of Xi' an Jiaotong University approved this study. All patients provided written informed consent.

\section{Serum Multiple Cytokine-Profiling Analysis}

Fasting blood $(5 \mathrm{~mL})$ was collected from each participant before the onset of SLIT, and processed and stored as described previously. ${ }^{13}$ A human cytokine standard 31-Plex assay kit (Bio-Rad) was utilized to detect 31 cytokines - BAFF, CCL2, CCL3, CCL4, CCL7, CCL11, CXCL9, CXCL10, CXCL12, GM-CSF, IFN $\gamma$, IL1 $\alpha$, IL1 $\beta$, IL2, IL2R $\alpha$, IL4, IL5, IL6, IL7, IL8, IL9, IL10, IL13, IL15, IL16, IL17, IL33, M-CSF, MIF, TNF $\alpha$, and TNF $\beta$ - based on the Luminex 200 system, and data interpretation was conducted as previously described. ${ }^{19}$ All samples were diluted at 1:3 with sample diluent, and data from the 31-Plex assays were transferred to an Excel spreadsheet for further analysis.

\section{Immunotherapy and Efficacy}

All patients were assigned to receive 3 years of standard SLIT, administered with standardized Dermatophagoides farina allergen drops (Wolwo Pharma Biotechnology). The entire SLIT course consists of an escalation phase and a maintainenance phase, and specific daily dosing schedules were conducted as our previous study described. During the SLIT, compliance education and follow-up were performed, medication score and total nasal symptom score (TNSS) evaluated via WeChat in the previous week and recorded weekly, and symptom and medication score (SMS) calculated as (TNSS + MS)/7, as previously described. ${ }^{13}$ At 1-year follow-up, early efficacy of SLIT was evaluated based on change in SMS: patients who attained a $30 \%$ reduction in SMS from baseline were defined as responders and the rest nonresponders as per our previous study. ${ }^{13}$

\section{Validation of Potential Cytokines}

Another independent cohort consisting of 98 AR patients treated with SLIT was used to confirm significantly distinctive cytokines in the discovery cohort. At 1-year follow-up, 80 patients had completed the treatment schedule: 50 responders and 30 nonresponders. Serum samples were harvested from all patients pre-SLIT, but only 40 patients provided serum specimens at 1 year post-SLIT. Concentrations of cytokines were measured using commercial ELISA kits and changes between pre-SLIT and post-SLIT levels assessed.

\section{Statistical Analysis}

Quantitative variables are shown as means $\pm \mathrm{SD}$ or median and interquartile range, and Student's $t$-tests or KruskalWallis $H$ tests were used for comparisons between two groups. Categorical variables are shown as numbers and percentages, and the $?^{2}$ test was used for comparison of same. Statistical analysis was performed with SPSS 23.0, and figures were constructed with GraphPad Prism 7.0. Receiver-operating characteristic (ROC) curves were constructed to evaluate cytokines with potential for predicting the efficacy of SLIT. $P<0.05$ was considered statistically significant. 
Table I Demographic and clinical characteristics of all AR patients

\begin{tabular}{|l|c|c|c|}
\hline & Responders (n=35) & Nonresponders (n=25) & $P$ \\
\hline Age (years), median (range) & $28(23-38)$ & $29.0(24-33)$ & 0.423 \\
Male, $\mathrm{n}(\%)$ & $17(48.6)$ & $13(52.0)$ & 1 \\
BMI (kg/m²) & $22.6(21.6-23.6)$ & $22.7(21.9-24.1)$ & 0.620 \\
Asthma, $\mathrm{n}(\%)$ & $9(25.7)$ & $9(36.0)$ & 0.409 \\
Serum total lgE (IU/mL) & $313.3(236.9-389.5)$ & $49.7(228.3-359.7)$ & 0.675 \\
Serum-specific IgE (IU/mL) & $41.3(30.2-62.5)$ & $9.0(7-10)$ & 0.923 \\
TNSS, median (range) & $9(8-10)$ & $7.0(5.0-8.0)$ & 0.170 \\
VAS score, median (range) & $7(6-9)$ & 0.319 \\
\hline
\end{tabular}

Abbreviations: AR, allergic rhinitis; BMI, body-mass index; TNSS, total nasal symptom score; VAS, visual analogue scale.

\section{Results}

\section{Patient Characteristics}

At 1-year follow-up, 60 AR patients had completed the 1-year visit schedule and provided complete follow-up data. In sum, 35 patients were categorized as responders and 25 as nonresponders. The main demographic and clinical

Table 2 Serum cytokine concentrations in the two groups $(\mathrm{pg} / \mathrm{mL})$

\begin{tabular}{|c|c|c|c|}
\hline Cytokines & Responders $(n=35)$ & Nonresponders $(n=25)$ & $P$ \\
\hline BAFF & $6,404.1(5,6 \mid 4.4-7,958.5)$ & $5,039.5(4,224.4-6,040.3)$ & 0.001 \\
\hline CCL2 & $28.0(19.8-44.2)$ & $44.9(27.2-120.2)$ & 0.005 \\
\hline CCL3 & $6.5(3.7-6.5)$ & $7.4(5.1-13.2)$ & 0.066 \\
\hline CCL4 & $145.2(130.2-168.5)$ & $155.5(135.0-196.6)$ & 0.215 \\
\hline CCL7 & $0.3(0.2-0.4)$ & $0.8(0.3-5.7)$ & 0.018 \\
\hline CCLII & $46.3(31.7-65.5)$ & $32.8(23.9-49.9)$ & 0.025 \\
\hline CXCL9 & 144.8 (| I0.7-232.9) & 159.5 (I28.2-239.5) & 0.653 \\
\hline CXCLIO & $284.5(231.3-472.3)$ & $381.2(282.9-431.3)$ & 0.910 \\
\hline CXCLI2 & $772.0(644.3-966.7)$ & $834.6(742.8-1076.0)$ & 0.148 \\
\hline GM-CSF & $0.7(0.4-1.5)$ & $1.0(0.4-1.8)$ & 0.132 \\
\hline $\mathrm{IFN} \gamma$ & $3.7(3.0-4.7)$ & $6.1(3.7-7.4)$ & 0.006 \\
\hline ILI $\alpha$ & $8.7(5.0-14.0)$ & $10.1(6.5-15.2)$ & 0.636 \\
\hline ILI $\beta$ & $2.0(1.4-2.6)$ & $2.0(1.6-2.8)$ & 0.900 \\
\hline IL2 & $0.6(0.4-0.6)$ & $0.6(0.4-0.8)$ & 0.130 \\
\hline IL2R $\alpha$ & $69.3(50.1-95.8)$ & $61.5(52.4-89.2)$ & 0.722 \\
\hline IL4 & $2.0(1.7-2.3)$ & $1.7(1.4-2.3)$ & 0.222 \\
\hline IL5 & $2.3(1.0-2.6)$ & $2.4(1.2-3.1)$ & 0.449 \\
\hline IL6 & $0.3(0.3-0.7)$ & $0.6(0.3-1.0)$ & 0.130 \\
\hline IL7 & $3.8(2.6-6.2)$ & $3.8(2.6-6.2)$ & 0.881 \\
\hline IL8 & $43.0(19.5-118.2)$ & $79.8(54.4-131.7)$ & 0.041 \\
\hline IL9 & $238.4(220.2-249.8)$ & $241.8(227.7-250.8)$ & 0.266 \\
\hline ILIO & $1.0(0.4-1.2)$ & $2.0(1.5-3.6)$ & 0.004 \\
\hline ILI3 & $1.7(1.0-2.9)$ & $1.5(0.9-2.2)$ & 0.121 \\
\hline ILI5 & $32.6(25.4-45.4)$ & $32.7(20.5-52.8)$ & 0.385 \\
\hline ILI6 & $79.8(5 \mid .2-110.4)$ & II $3.4(66.6-184.3)$ & 0.020 \\
\hline ILI7 & $5.5(4.8-6.7)$ & $5.9(4.8-7.0)$ & 0.537 \\
\hline IL33 & $67.5(34.0-103.8)$ & $142.5(54.7-\mid 78.1)$ & 0.001 \\
\hline M-CSF & $21.8(17.6-30.1)$ & $23.1(20.3-37.7)$ & 0.216 \\
\hline MIF & $1,131.0(681.3-1,565.4)$ & $1,420.0(785.6-1,822.0)$ & 0.335 \\
\hline $\mathrm{TNF} \alpha$ & $16.5(|4|-2 \mid .2)$. & $19.4(14.1-24.9)$ & 0.412 \\
\hline TNF $\beta$ & $226.0(208.2-240.0)$ & $231.6(210.6-238.9)$ & 0.644 \\
\hline
\end{tabular}

Abbreviations: BAFF, B cell activating factor; CCL, CC motif chemokine ligand; CXCL, C-X-C motif chemokine ligand; GM-CSF, granulocyte monocytecolony stimulating factor; IFN, interferon; IL, interleukin; M-CSF, macrophage colony stimulating factor; MIF, macrophage migration inhibitory factor; TNF, tumor necrosis factor. 
A

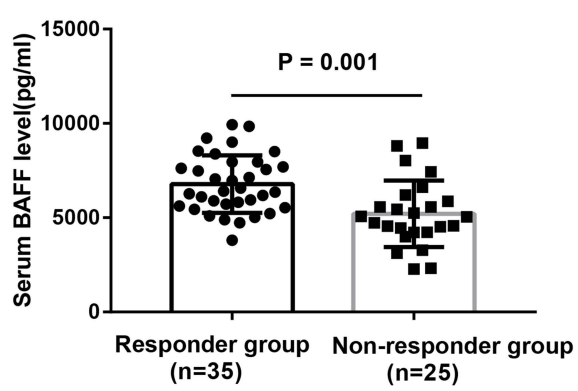

D

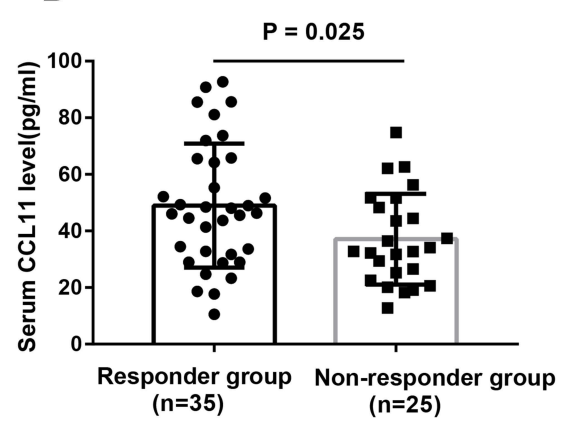

G

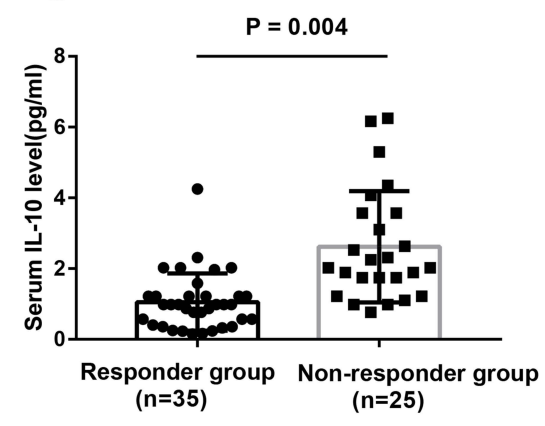

B

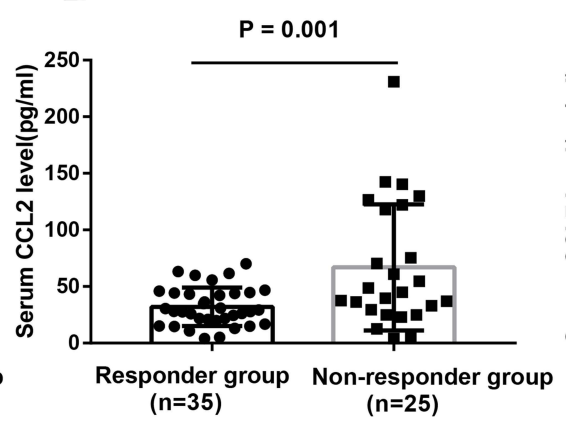

E

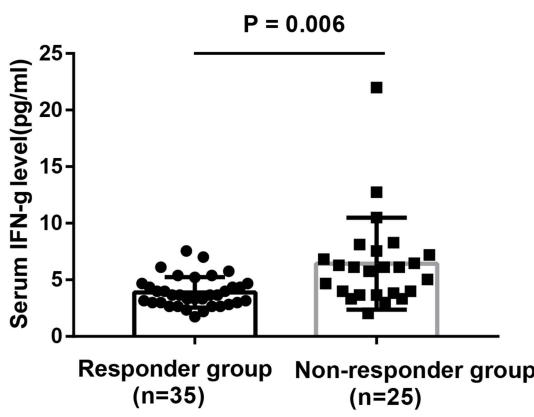

H

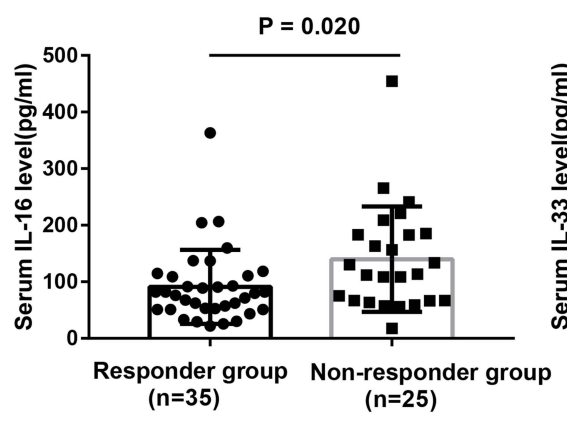

C

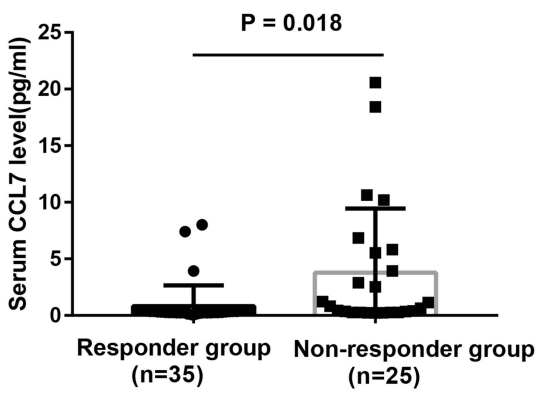

F

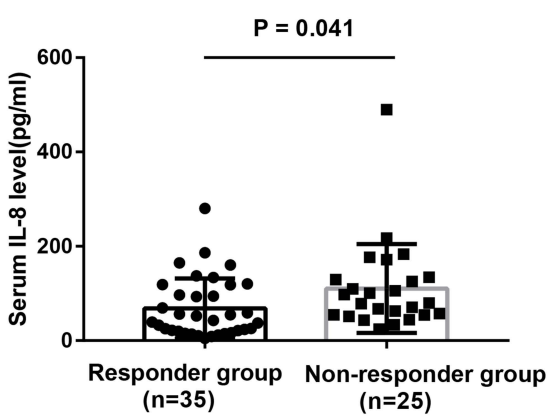

I

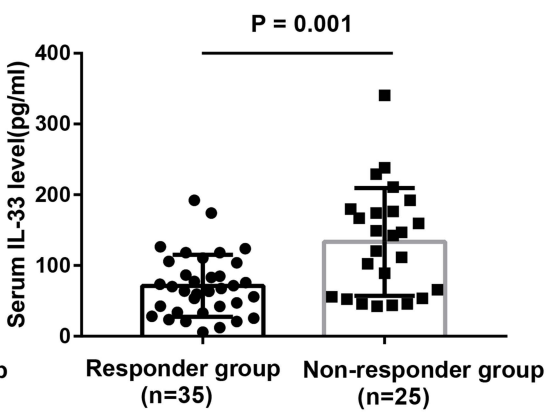

Figure I Serum levels of 9 differential cytokines between responder group and nonresponder group (A-I).

Abbreviations: BAFF, B cell activating factor; CCL, CC motif chemokine ligand; IFN, interferon; IL, interleukin.

characteristics of all patients in the prospective cohort are shown in Table 1. No significant differences in age, sex, BMI, asthma, serum total and specific IgE levels, TNSS, or VAS scores were observed.

\section{Cytokine Profiling Differs Between Responders and Nonresponders}

Levels of 31 cytokines in responders and nonresponders are displayed in Table 2. As shown in Figure 1, nine cytokines were significantly different between the two groups, with responders having higher concentrations of BAFF and CCL11 and lower levels of CCL2, CCL7, IFN $\gamma$, IL8, IL10, IL16, and IL33 than nonresponders $(P<0.05)$. ROC curves highlighted that serum BAFF, IFN $\gamma$, IL10, and IL33 exhibited strong ability for predicting the efficacy of SLIT (area under the curve $<0.7, P<0.05$; Figure 2), and detailed parameters are given in Table 3 .

\section{Validation of Potential Cytokines}

In order to validate the predictive abilities of BAFF, IFN $\gamma$, IL10, and IL33, another independent cohort was studied. Table 4 presents the demographic and clinical characteristics of all participants. As shown in Figure 3, responders 
A

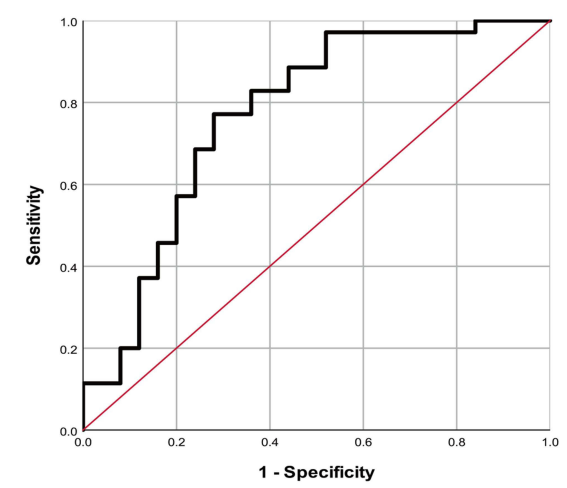

D

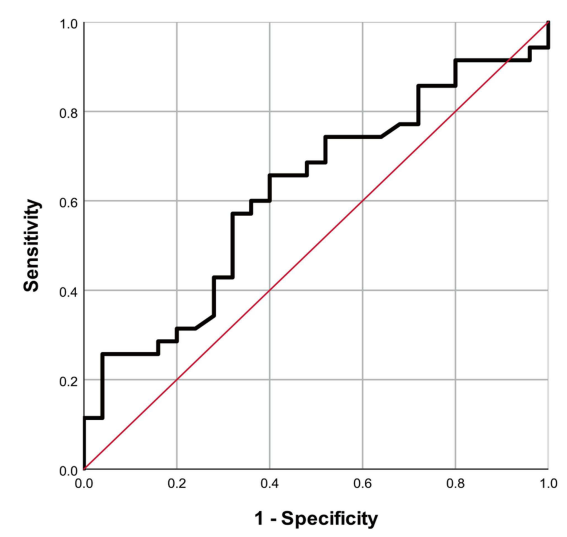

G

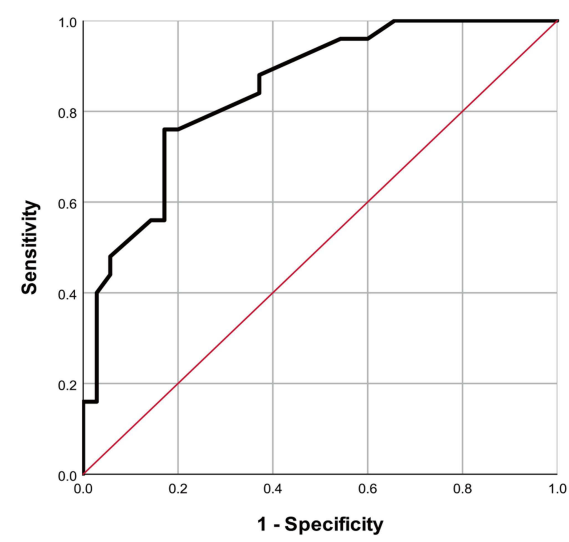

B

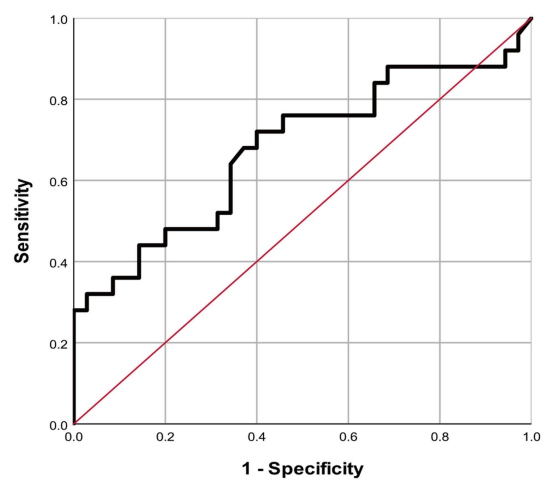

E

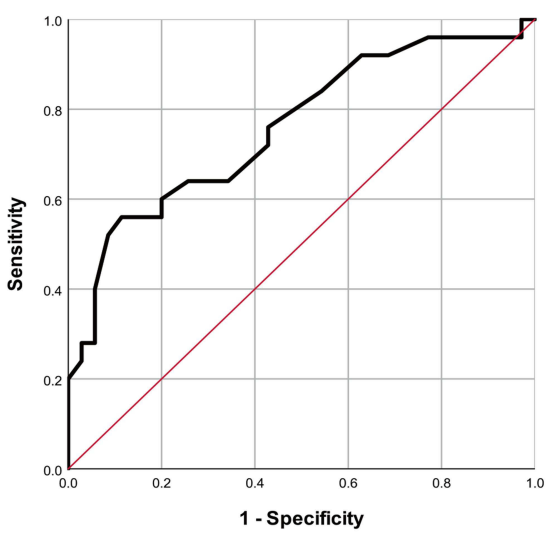

H

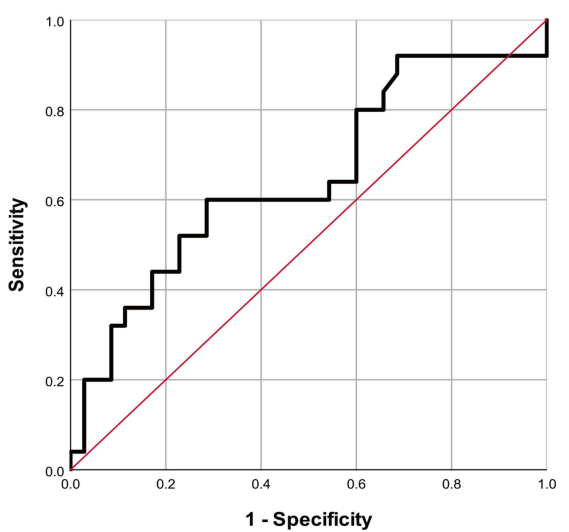

C

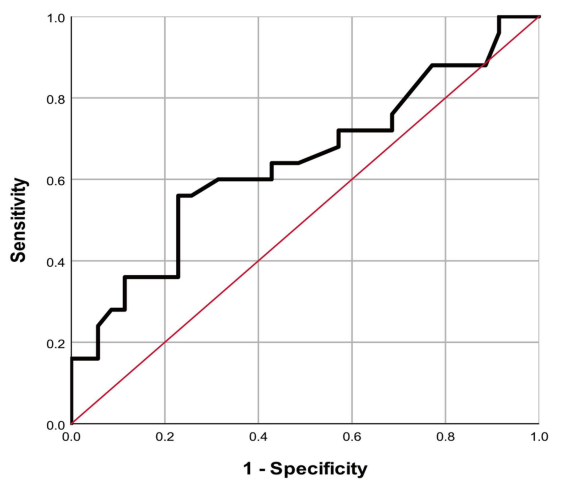

F

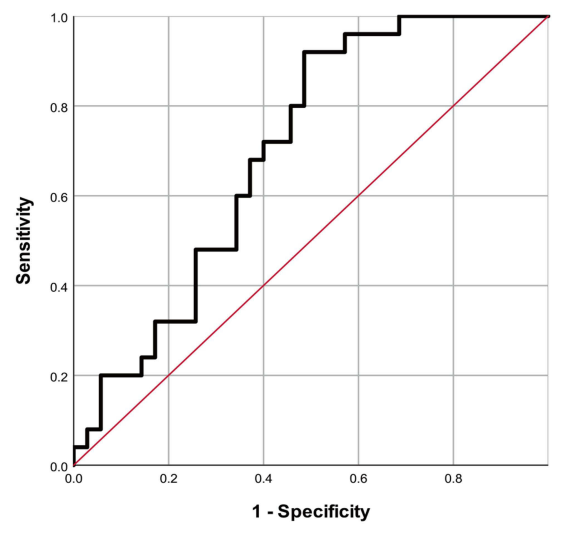

I

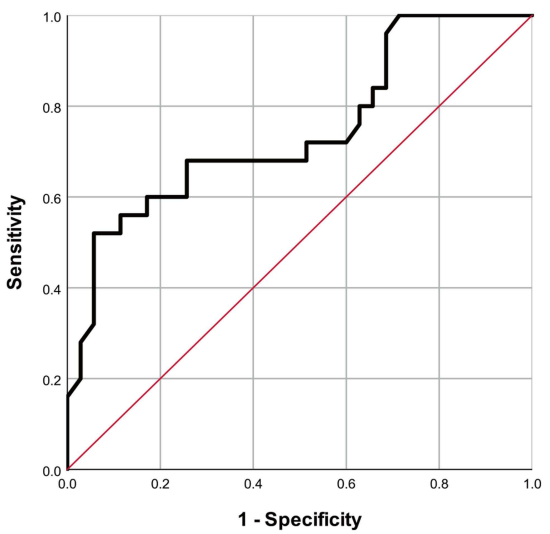

Figure 2 ROC curves of nine cytokines in predicting the efficacy of SLIT. (A) BAFF; (B) CCL2; (C) CCL7; (D) CCLI I; (E) IFN $\gamma ;$ (F) IL8; (G) ILI0; (H) ILI6; (I) IL33. Abbreviations: ROC, receiver-operating characteristic; SLIT, sublingual immunotherapy.

exhibited higher concentrations of IL10 and IL33 than nonresponders $(P<0.05)$, but serum BAFF and IFN $\gamma$ levels were not significantly different between the two groups. Moreover, respondes exhibited significantly higher IL10 levels and lower IL33 post-SLIT than pre-SLIT $(P<0.05)$, but no significant difference was found in nonresponders. ROC curves revealed that serum IL10 and IL33 presented reliable accuracy for predicting the efficacy of SLIT in the validation cohort (Figure 4 and Table 5). 
Table 3 ROC results for ytokines in terms of SLIT efficacy $(\mathrm{pg} / \mathrm{mL})$

\begin{tabular}{|l|l|l|c|l|c|}
\hline & AUC $(\mathbf{9 5} \% \mathbf{C I})$ & $\boldsymbol{P}$ & Cutoff & Sensitivity & Specificity \\
\hline BAFF & $0.770(0.642-0.899)$ & $<0.001$ & 5591.1 & 0.77 I & 0.680 \\
CCL2 & $0.678(0.533-0.822)$ & 0.020 & 35.6 & 0.680 & 0.629 \\
CCL7 & $0.642(0.495-0.788)$ & 0.075 & 0.6 & 0.560 & 0.77 I \\
CCLII & $0.619(0.476-0.763)$ & 0.073 & 44.5 & 0.571 & 0.680 \\
IFN & $0.760(0.634-0.886)$ & 0.001 & 5.6 & 0.560 & 0.886 \\
IL8 & $0.699(0.569-0.830)$ & 0.009 & 43.1 & 0.920 & 0.514 \\
ILI0 & $0.847(0.751-0.944)$ & $<0.001$ & 1.7 & 0.760 & 0.829 \\
ILI6 & $0.647(0.502-0.793)$ & 0.053 & 100.7 & 0.600 & 0.714 \\
IL33 & $0.745(0.615-0.875)$ & 0.001 & 134.5 & 0.520 & 0.943 \\
\hline
\end{tabular}

Abbreviations: ROC, receiver-operating characteristic; SLIT, sublingual immunotherapy; AUC, area under the curve.

\section{Discussion}

The present study explored multiple-cytokine profiling in the sera of AR patients treated with SLIT and evaluated associations with SLIT effectiveness. We firstly observed that cytokine levels were significantly different between responders and nonresponders, and that serum BAFF, IFN $\gamma$, IL10, and IL33 might be associated with the efficacy of SLIT. Further validation results revealed that serum IL10 and IL33 were elevated in nonresponders in the validation cohort, and patients in the responder group exhibited significantly higher IL10 and lower IL33 post-SLIT than pre-SLIT. Taken together, our observations indicate that serum IL10 and IL33 might serve as potential biomarkers for objectively predicting the efficacy of SLIT and contribute to its therapeutic mechanisms.

IL10 is an anti-inflammatory cytokine that acts as a pivotal immunoregulatory molecule and is involved in inflammatory and allergic diseases. ${ }^{20,21}$ Previous studies have demonstrated that IL10 levels are dysregulated in nasal lavage fluid, nasal mucosa, and peripheral blood of AR patients and animal models and associated with the occurrence and development of AR. ${ }^{22-24}$ Boonpiyathad et $\mathrm{al}^{25}$ reported that IL10 activated innate lymphoid cells and the number of innate IL $10^{+}$lymphoid cells was associated with the efficacy of AIT in house dust-mite AR patients. A recent study found that serum IL10 levels were enhanced in AR patients who were treated with SLIT and vitamin D supplementation, which implied that IL10 expression might be involved in the development of immunotolerance. ${ }^{26}$ In the present study, serum IL10 levels were strongly linked with the effectiveness of SLIT and IL10 concentrations significantly enhanced in responders after 1 year's SLIT, but did not change in nonresponders between pre-SLIT and post-SLIT, in line with most prior conclusions. ${ }^{25,26}$ Development of immunotolerance is a critical process in immunotherapy. The induction of $\mathrm{T}_{\text {reg }}$ and $\mathrm{B}_{\text {reg }}$ cells have been proven to be modified during AIT and correlated with its clinical efficacy. ${ }^{27-29}$ An elevated number of $T_{\text {regs }}$ and $B_{\text {regs }}$ promotes the secretion of IL10 to dampen the $T_{h} 2$ phenotype and reinforce the $T_{h} 1$ phenotype. $^{30-32}$ Moreover, accumulating evidence has shown that IL10-producing $\mathrm{T}_{\text {reg, }}, \mathrm{B}_{\text {reg, }}$ and group 2 innate lymphoid (ILC2) cell counts correlate inversely with symptom severity after AIT. ${ }^{33,34}$ These observations suggest that serum levels might serve as an indicator of the success of AIT; however, the underlying mechanisms remain unclear.

Table 4 Demographic and clinical characteristics of subjects in the validation cohort

\begin{tabular}{|l|c|c|c|}
\hline & Responders (n=50) & Nonresponders (n=30) & $P$ \\
\hline Age, years & $28(24-33)$ & $30(25-37)$ & 0.307 \\
Male, $\mathrm{n}(\%)$ & $23(46)$ & $19(63.3)$ & 0.927 \\
BMI $\left(\mathrm{kg} / \mathrm{m}^{2}\right)$ & $22.1(21.2-23.2)$ & $22.0(21.1-23.0)$ & 0.618 \\
Asthma, $\mathrm{n}(\%)$ & $12(40)$ & 0.327 \\
Serum total IgE (IU/mL) & $291.7(218.8-372.5)$ & $279.4(219.5-372.1)$ & 0.782 \\
Serum-specific lgE (IU/mL) & $33.6(15.8-60.9)$ & $36.0(27.8-54.6)$ & 0.423 \\
TNSS, median (range) & $9(6-10)$ & $6(5-8)$ & 0.213 \\
VAS score, median (range) & $7(5-8)$ & $5(4-7)$ & 0.975 \\
\hline
\end{tabular}

Abbreviations: BMI, body-mass index; TNSS, total nasal symptom score; VAS, visual analogue scale. 
A

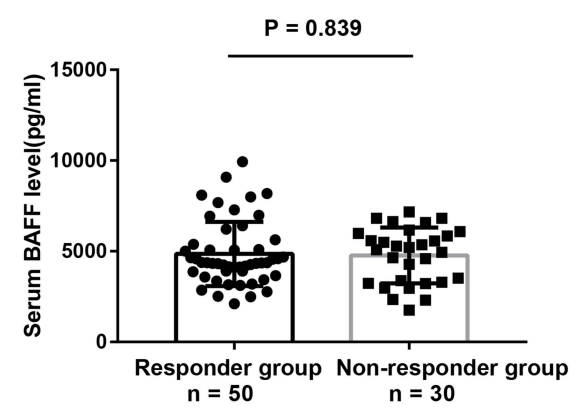

D

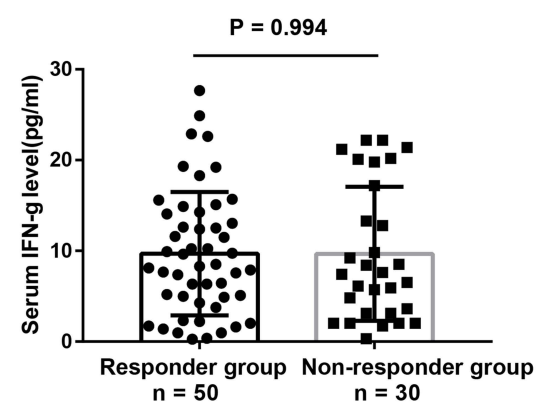

G

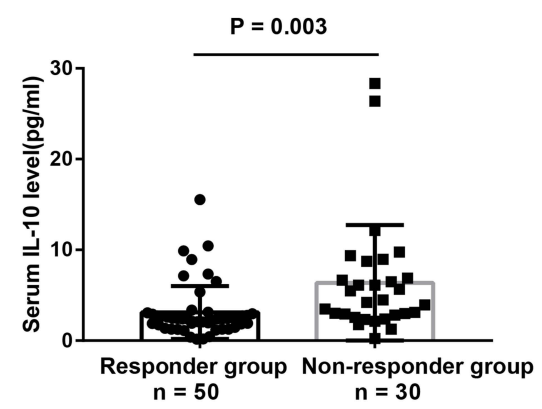

J

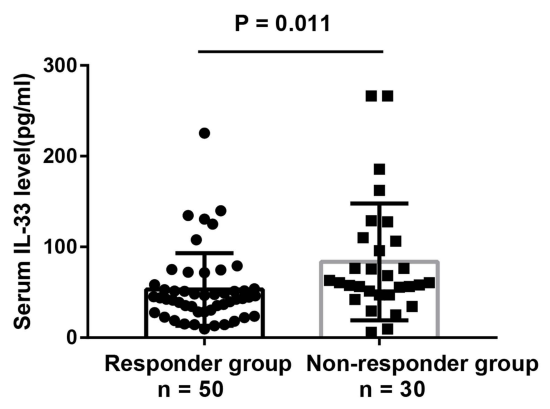

B

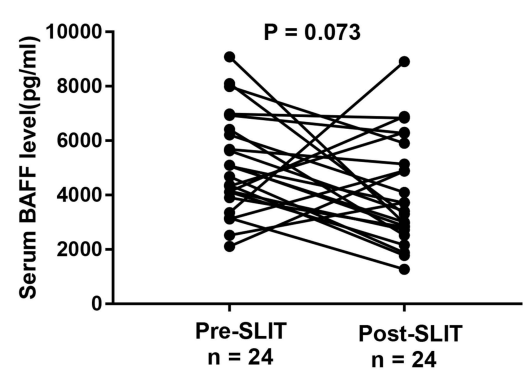

E

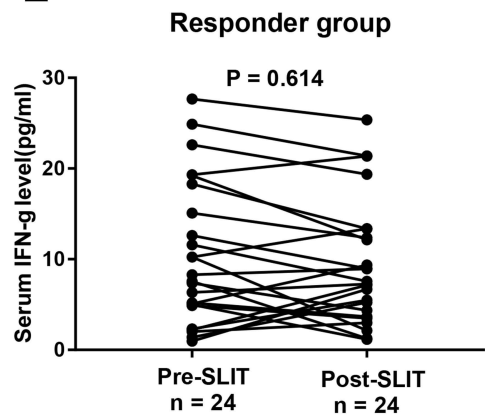

H

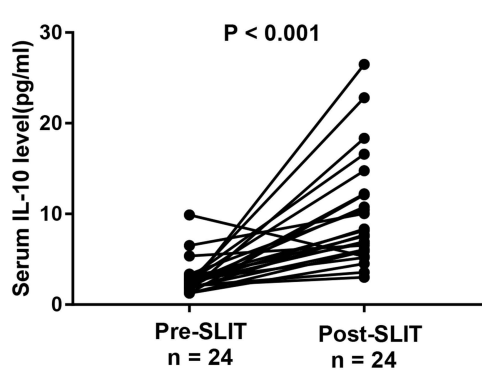

K

Responder group

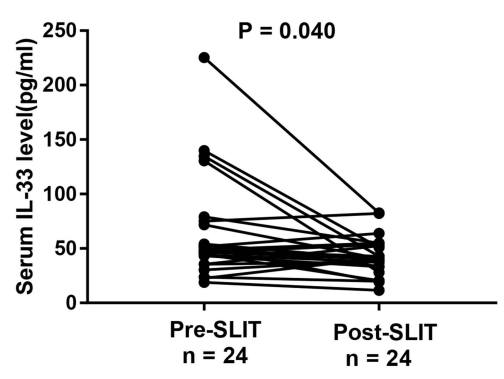

C Non-responder group $P=0.134$

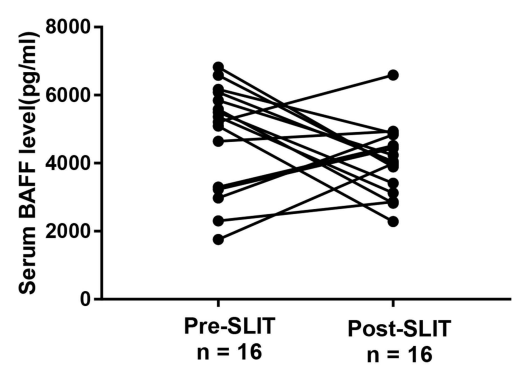

F Non-responder group

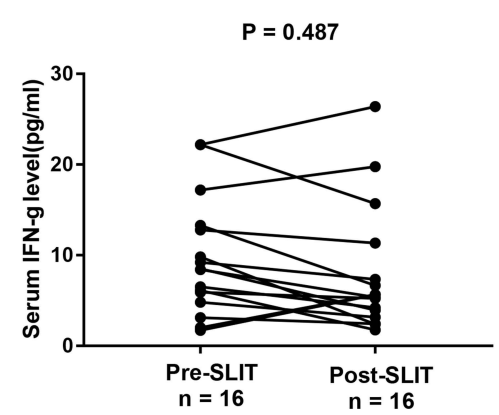

I Non-responder group

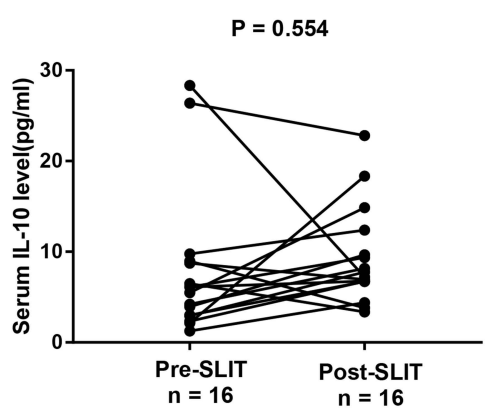

L Non-responder group $P=0.252$

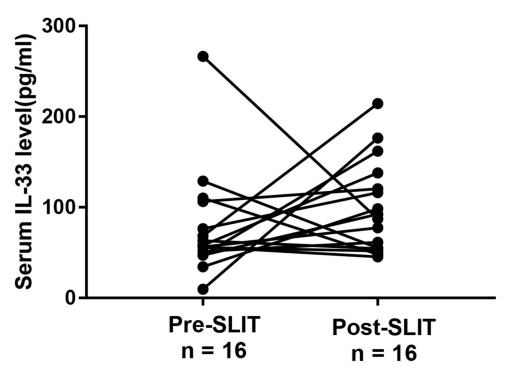

Figure 3 Serum levels of four potential cytokines in validation cohort. (A-C) BAFF; (D-F) IFN $\gamma ;$ (H and I) ILI0; (J-L) IL33. Abbreviation: SLIT, sublingual immunotherapy.

Another important finding in the present study was that serum IL33 levels were associated with the effectiveness of SLIT and that concentrations were reduced when patients responded to SLIT. IL33 is known to be an epithelial alarmin cytokine that is released upon external irritants, contributes to activation of ILC2 and $\mathrm{T}_{\mathrm{h}} 2$ cells, and involved in mucosa 
A

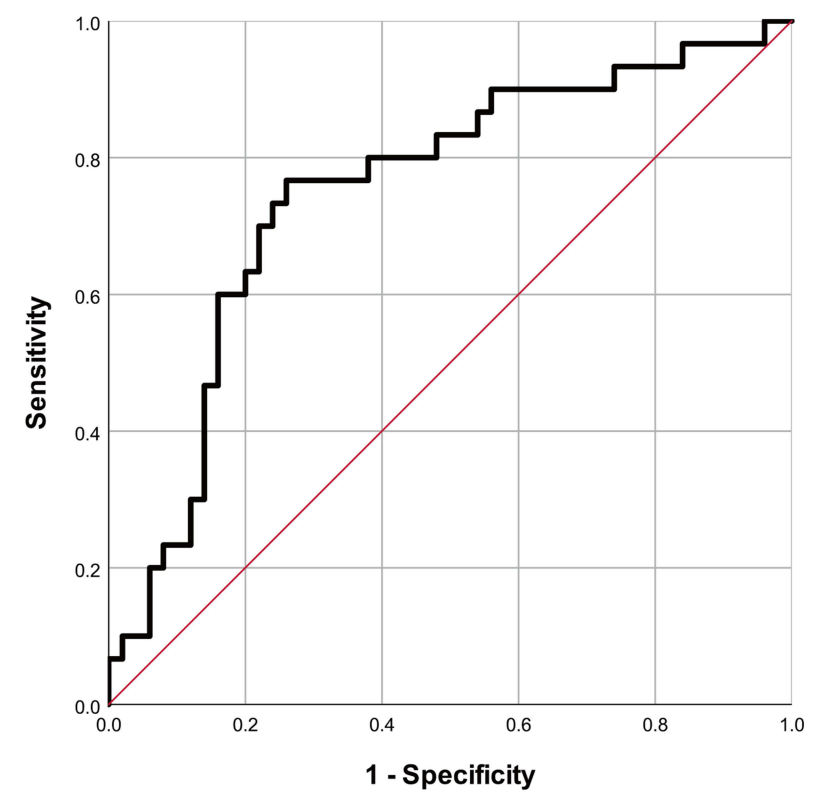

B

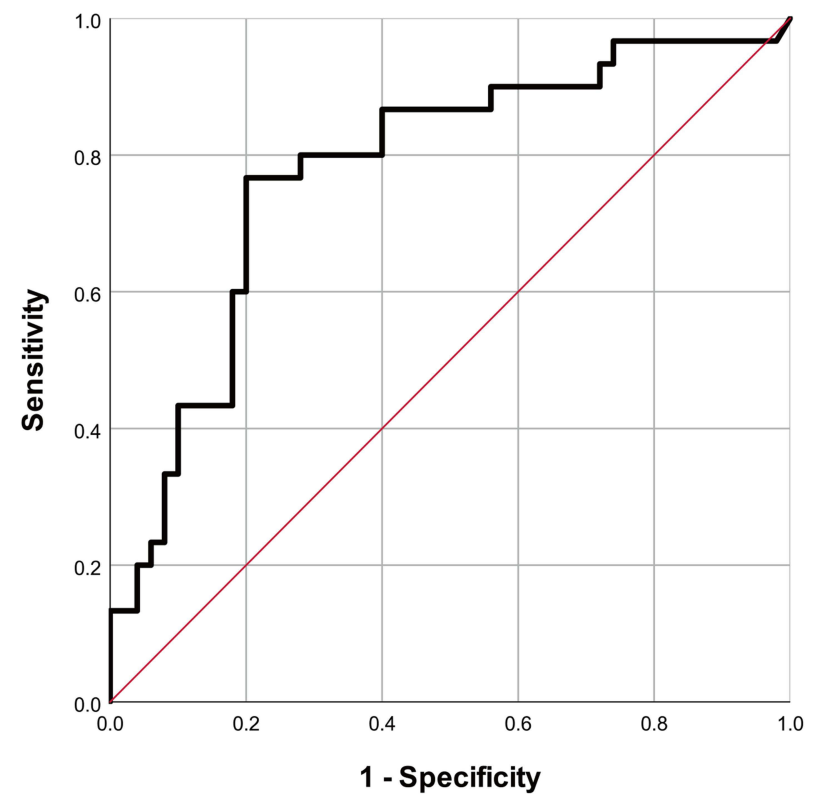

Figure 4 ROC curves of serum ILI0 (A) and IL33 (B) in predicting the efficacy of SLIT in the validation cohort. Abbreviations: ROC, receiver-operating characteristic; SLIT, sublingual immunotherapy.

eosinophilia and immunoresponse. ${ }^{35}$ Studies have reported a crucial role of IL33 in the inflammatory response and tissue remodeling in asthma and AR. ${ }^{36,37}$ A recent publication found that serum IL33 and its receptor ST2 were lower in AR patients than healthy controls and that SLIT enhanced IL33 expression, but did not change ST2 levels. ${ }^{38}$ However, Wang et $\mathrm{al}^{39}$ proved that IL33 levels were decreased during SLIT in the nasal lavage of AR children and positively correlated with increased IL10 expression. In this study, we found that serum IL33 levels were closely associated with the efficacy of SLIT in AR patients and reduced after 1 year's treatment, in accordance with Wang et al's conclusion. Accordingly, IL33 has been found to be an essential promoter in $\mathrm{T}_{\mathrm{h}} 2$-based inflammation. ${ }^{40}$ High concentrations of IL33 can activate its ST2 receptor and facilitate the production of $\mathrm{T}_{\mathrm{h}} 2$ cytokines and IgE, then aggravate eosinophil recruitment and airway hyperreactivity, resulting in refractory inflammation in AR and a failure of SLIT. As such, we can speculate that IL33 might play a decisive role in the underlying therapeutic mechanism of SLIT and serum IL33 is a reliable biomarker for predicting the efficacy of SLIT in AR patients.

Although we observed that responders exhibited higher serum BAFF and lower IFN $\gamma$ levels than nonresponders in the discovery cohort, these tendencies were not found in the validation cohort. We speculate that these two cytokines might serve a limited role in the therapeutic effects of SLIT in AR patients. More studies are needed to clarify their functions in AR.

There are several limitations to this study. First, the sample is relatively small. All patients were from a single medical center and of the same ethnicity, which may have increased the risk of selection bias and limit generalization. Second, follow-up was relatively brief, and we evaluated only the early efficacy of SLIT. Associations between multiple-cytokine levels and more prolonged efficacy of SLIT are not clear.

Table 5 ROC analysis results for ILIO and IL33 in terms of SCIT efficacy (pg/mL)

\begin{tabular}{|l|l|l|l|l|l|}
\hline & AUC $(95 \%$ Cl) & P & Cutoff & Sensitivity & Specificity \\
\hline ILI0 & $0.750(0.636-0.864)$ & $<0.001$ & 2.8 & 0.767 & 0.740 \\
IL33 & $0.778(0.669-0.886)$ & $<0.001$ & 55.4 & 0.767 & 0.800 \\
\hline
\end{tabular}

Abbreviations: ROC, receiver-operating characteristic; SLIT, sublingual immunotherapy; AUC, area under the curve. 
In conclusion, we firstly confirmed that serum multiple-cytokine profiling was associated with the response to SLIT and that serum IL10 and IL33 might serve as novel biomarkers for early prediction of efficacy. We also found that serum IL10 and IL33 levels changed during SLIT in AR patients, which suggests that they were involved in the therapeutic mechanisms of SLIT. Further multicenter studies with a large samples and longer follow-up are needed to confirm our conclusions.

\section{Data Sharing}

The data used to support the observations of this study are available from the corresponding author upon request.

\section{Ethics}

This study was conducted in accordance with the recommendations of the Declaration of Helsinki. The Human Ethics Committee of the Second Affiliated Hospital of Xi'an Jiaotong University approved the study, and all participants provided informed consent.

\section{Funding}

This work was supported by the National Natural Science Foundation of China (62076198) and the General Projects of Shaanxi Provincial Key Research and Development Program (2020GXLH-Y005).

\section{Disclosure}

The authors declare that the research was conducted in the absence of any commercial or financial relationships that could be construed as a potential conflict of interest.

\section{References}

1. Ogulur I, Pat Y, Ardicli O, et al. Advances and highlights in biomarkers of allergic diseases. Allergy. 2021;76:3659-3686. doi:10.1111/all.15089

2. Zhang Y, Lan F, Zhang L. Advances and highlights in allergic rhinitis. Allergy. 2021;76:3383-3389. doi:10.1111/all.15044

3. Ren J, Pang W, Luo Y, et al. Impact of allergic rhinitis and asthma on COVID-19 infection, hospitalization and mortality. J Allergy Clin Immunol Pract. 2021. doi:10.1016/j.jaip.2021.10.049

4. Schuler IV CF, Montejo JM. Allergic rhinitis in children and adolescents. Immunol Allergy Clin North Am. 2021;41:613-625. doi:10.1016/j. iac.2021.07.010

5. Papadopoulos NG, Aggelides X, Stamataki S, Prokopakis E, Katotomichelakis M, Xepapadaki P. New concepts in pediatric rhinitis. Pediatr Allergy Immunol. 2021;32:635-646. doi:10.1111/pai.13454

6. Sultész M, Horváth A, Molnár D, et al. Prevalence of allergic rhinitis, related comorbidities and risk factors in schoolchildren. Allergy Asthma Clin Immunol. 2020;16:98. doi:10.1186/s13223-020-00495-1

7. Okubo K, Kurono Y, Ichimura K, et al. Japanese guidelines for allergic rhinitis 2020. Allergol Int. 2020;69:331-345. doi:10.1016/j.alit.2020.04.001

8. Li H, Chen S, Cheng L, et al. Chinese guideline on sublingual immunotherapy for allergic rhinitis and asthma. J Thorac Dis. 2019;11:4936-4950. doi: $10.21037 /$ jtd.2019.12.37

9. Licari A, Castagnoli R, Brambilla I, et al. Biomarkers of immunotherapy response in patients with allergic rhinitis. Expert Rev Clin Immunol. 2018;14:657-663. doi:10.1080/1744666X.2018.1504679

10. Liu Z, Lu H, Feng X, Hu L, Wang J, Yu H. Predictive methods for efficacy of house dust mite subcutaneous immunotherapy in allergic rhinitis patients: a prospective study in a Chinese population. Int Forum Allergy Rhinol. 2020;10:314-319. doi:10.1002/alr.22508

11. Gotoh M, Kaminuma O, Nakaya A, et al. Identification of biomarker sets for predicting the efficacy of sublingual immunotherapy against pollen-induced allergic rhinitis. Int Immunol. 2017;29:291-300. doi:10.1093/intimm/dxx034

12. Lam HY, Tergaonkar V, Ahn KS. Mechanisms of allergen-specific immunotherapy for allergic rhinitis and food allergies. Biosci Rep. $2020 ; 40$. doi:10.1042/BSR20200256

13. Zhu K, Xia C, Chen J, et al. Serum soluble ST2 correlated with symptom severity and clinical response of sublingual immunotherapy for house dust mite-induced allergic rhinitis patients. Mediators Inflamm. 2021;2021:5576596. doi:10.1155/2021/5576596

14. Kim JY, Rhee CS, Mun SJ, Cho SH, Lockey RF, Han DH. Early response of specific IgE can predict satisfaction with sublingual immunotherapy. Laryngoscope. 2020;131:467-472.

15. Xie S, Zhang H, Wang F, Xie Z, Jiang W, Gao K. Circulating MIF associated with disease severity and clinical response of sublingual immunotherapy in house dust mite-induced allergic rhinitis. Front Pharmacol. 2021;12:681724. doi:10.3389/fphar.2021.681724

16. Xie S, Jiang S, Zhang $\mathrm{H}$, et al. Prediction of sublingual immunotherapy efficacy in allergic rhinitis by serum metabolomics analysis. Int Immunopharmacol. 2021;90:107211. doi:10.1016/j.intimp.2020.107211

17. Joudi M, Farid Hosseini R, Khoshkhui M, et al. Effects of serum vitamin D and efficacy of subcutaneous immunotherapy in adult patients with allergic rhinitis. Allergy Asthma Immunol Res. 2019;11:885-893. doi:10.4168/aair.2019.11.6.885

18. Brozek JL, Bousquet J, Agache I, et al. Allergic Rhinitis and its Impact on Asthma (ARIA) guidelines-2016 revision. J Allergy Clin Immunol. 2017;140:950-958. doi:10.1016/j.jaci.2017.03.050

19. Owaki T, Imai K, Miki R, et al. Multiple cytokine analysis in gastroschisis: association with adverse outcomes including fetal brain damage. Cytokine. 2021;138:155406. doi:10.1016/j.cyto.2020.155406 
20. Oka A, Kidoguchi M, Kariya S, et al. Role of salivary microbiome in IL-10 production and efficacy of sublingual immunotherapy. Allergy. 2021;76:2617-2620. doi:10.1111/all.14858

21. Erkan K, Bozkurt MK, Artaç H, et al. The role of regulatory T cells in allergic rhinitis and their correlation with IL-10, IL-17 and neopterin levels in serum and nasal lavage fluid. Eur Arch Otorhinolaryngol. 2020;277:1109-1114. doi:10.1007/s00405-020-05811-4

22. Kang MG, Han SW, Kang HR, Hong SJ, Kim DH, Choi JH. Probiotic NVP-1703 alleviates allergic rhinitis by inducing IL-10 expression: a four-week clinical trial. Nutrients. 2020;12:1427. doi:10.3390/nu12051427

23. Zissler UM, Jakwerth CA, Guerth FM, et al. Early IL-10 producing B-cells and coinciding Th/Tr17 shifts during three year grass-pollen AIT. EBioMedicine. 2018;36:475-488. doi:10.1016/j.ebiom.2018.09.016

24. Shao JB, Luo XQ, Wu YJ, et al. Histone deacetylase 11 inhibits interleukin 10 in B cells of subjects with allergic rhinitis. Int Forum Allergy Rhinol. 2018;8:1274-1283. doi:10.1002/alr.22171

25. Boonpiyathad T, Tantilipikorn P, Ruxrungtham K, et al. IL-10-producing innate lymphoid cells increased in patients with house dust mite allergic rhinitis following immunotherapy. J Allergy Clin Immunol. 2021;147:1507-1510.e1508. doi:10.1016/j.jaci.2020.10.029

26. El-Maghraby HM, Rabie RA. Serum level of IL 10 is significantly increased in allergic rhinitis patients on subcutaneous immunotherapy and vitamin D supplementation. Egypt J Immunol. 2019;26:87-93.

27. Caruso M, Cibella F, Emma R, et al. Basophil biomarkers as useful predictors for sublingual immunotherapy in allergic rhinitis. Int Immunopharmacol. 2018;60:50-58.

28. Sharif H, Acharya S, Dhondalay GKR, et al. Altered chromatin landscape in circulating T follicular helper and regulatory cells following grass pollen subcutaneous and sublingual immunotherapy. J Allergy Clin Immunol. 2021;147:663-676. doi:10.1016/j.jaci.2020.10.035

29. Tanaka Y, Nagashima H, Bando K, et al. Oral CD103(-)CD11b(+) classical dendritic cells present sublingual antigen and induce Foxp3(+) regulatory T cells in draining lymph nodes. Mucosal Immunol. 2017;10:79-90. doi:10.1038/mi.2016.46

30. Shan M, Carrillo J, Yeste A, et al. Secreted IgD amplifies humoral T helper 2 cell responses by binding basophils via galectin-9 and CD44. Immunity. 2018;49:709-724.e708. doi:10.1016/j.immuni.2018.08.013

31. Boonpiyathad T, Sokolowska M, Morita H, et al. Der p 1-specific regulatory T-cell response during house dust mite allergen immunotherapy. Allergy. 2019;74:976-985. doi:10.1111/all.13684

32. van de Veen W, Wirz OF, Globinska A, Akdis M. Novel mechanisms in immune tolerance to allergens during natural allergen exposure and allergen-specific immunotherapy. Curr Opin Immunol. 2017;48:74-81. doi:10.1016/j.coi.2017.08.012

33. Golebski K, Layhadi JA, Sahiner U, et al. Induction of IL-10-producing type 2 innate lymphoid cells by allergen immunotherapy is associated with clinical response. Immunity. 2021;54:291-307.e297. doi:10.1016/j.immuni.2020.12.013

34. Shamji MH, Layhadi JA, Sharif H, Penagos M, Durham SR. Immunological responses and biomarkers for allergen-specific immunotherapy against inhaled allergens. J Allergy Clin Immunol Pract. 2021;9:1769-1778. doi:10.1016/j.jaip.2021.03.029

35. Pointner L, Bethanis A, Thaler M, et al. Initiating pollen sensitization - complex source, complex mechanisms. Clin Transl Allergy. $2020 ; 10: 36$. doi:10.1186/s13601-020-00341-y

36. Lee HY, Rhee CK, Kang JY, et al. Blockade of IL-33/ST2 ameliorates airway inflammation in a murine model of allergic asthma. Exp Lung Res. 2014;40:66-76. doi:10.3109/01902148.2013.870261

37. Haccuria A, Van Muylem A, Malinovschi A, et al. Increased expression of IL-33 is found in the lower airways of patients with seasonal allergic rhinitis and is not related to natural allergen exposure. Clin Exp Allergy. 2021;51:845-848. doi:10.1111/cea.13819

38. Glück J, Rymarczyk B, Jura-Szołtys E, Rogala B. Serum levels of interleukin 33 and its receptor ST2 in patients treated with subcutaneous allergen immunotherapy in intermittent allergic rhinitis. Cent Eur J Immunol. 2019;44:214-217. doi:10.5114/ceji.2019.87075

39. Wang Y, Li C, Xu Y, et al. Sublingual immunotherapy decreases expression of interleukin-33 in children with allergic rhinitis. Indian J Pediatr. 2018;85:872-876. doi:10.1007/s12098-018-2703-3

40. Ketelaar ME, Westerlaken-van Ginkel CD, Nawijn MC, Ej Dubois A, Koppelman GH. IL-1RL1a serum levels and IL1RL1 SNPs in the prediction of food allergy. Clin Exp Allergy. 2020;51:614.

Journal of Inflammation Research

\section{Publish your work in this journal}

The Journal of Inflammation Research is an international, peer-reviewed open-access journal that welcomes laboratory and clinical findings on the molecular basis, cell biology and pharmacology of inflammation including original research, reviews, symposium reports, hypothesis formation and commentaries on: acute/chronic inflammation; mediators of inflammation; cellular processes; molecular mechanisms; pharmacology and novel anti-inflammatory drugs; clinical conditions involving inflammation. The manuscript management system is completely online and includes a very quick and fair peer-review system. Visit http://www.dovepress.com/testimonials.php to read real quotes from published authors.

Submit your manuscript here: https://www.dovepress.com/journal-of-inflammation-research-journal 